\title{
Distribuição da mortalidade por cânceres ginecológicos no estado da Paraíba
}

\author{
Girleide Santos do Nascimento (D), Kelvyn Kennedy de Figueiredo Silva (iD \& \\ Bruna Braga Dantas $(\mathbb{C}$
}

Universidade Federal de Campina Grande, Centro de Educação e Saúde, Unidade Acadêmica de Saúde, Sítio Olho D’água da Bica, Zona Rural 58175-000, Cuité, Paraíba, Brasil.

E-mail: girleidesantos.picui8@gmail.com, brunabdantas@gmail.com
Nascimento G.S., Silva K.K.F. \& Dantas B.B. (2021) Distribuição da mortalidade por cânceres ginecológicos no estado da Paraíba. Pesquisa e Ensino em Ciências Exatas e da Natureza, 5(edição especial 1): e1629. http://dx.doi.org/10.29215/pecen.v5i0.1629

Responsabilidade editorial: Fagner Carvalho Leite. Recebido: 04 dezembro 2020. Aceito: 14 janeiro 2021. Publicado: 03 fevereiro 2021.

Resumo: Nas mulheres, os cânceres ginecológicos (CG) estão entre as enfermidades mais comuns, tornando-se problema de saúde mundial. Objetivou-se analisar a distribuição do perfil de mortalidade por CG em mesorregiões da Paraíba, durante o período de 1989 a 2018. Trata-se de uma abordagem ecológica, de série temporal, quantitativa e espacial. Os dados foram extraídos do atlas de mortalidade on-line do Instituto Nacional de Câncer José Alencar Gomes da Silva (INCA). A média de óbitos por CG na Paraíba foi equivalente a 1145.5, com maior número de mortes na mesorregião da Mata Paraibana (2056) e no Agreste Paraibano (1358). Todas as mesorregiões apresentaram um crescimento contínuo da média de óbitos ao longo dos anos, sendo maior aumento de óbitos na Borborema (4.5x). Quanto aos tipos de CG, observou-se que a maior média de mortes foi para câncer de colo de útero (227.8) na Mata Paraibana e a menor média de mortes ocorreu na região da Borborema, para câncer de vagina (1.0). Em todas as regiões, foi mais frequente mortes por câncer de útero, seguido de útero Sem Outra Especificação (SOE), ovário, corpo do útero, outros órgãos genitais, vulva e vagina. Conclui-se que há uma crescente mortalidade por CG em toda a Paraíba, com uma heterogeneidade entre regiões e entre tipos de cânceres.

Palavras chave: Epidemiologia, mortalidade, saúde da mulher, neoplasia por genitais femininas.

Distribution of mortality by gynecological cancers in the state of Paraíba

Abstract: In women, gynecological cancers (CG) are among the most common diseases, becoming a global health problem. The objective was to analyze the distribution of the mortality profile for GC in mesoregions of Paraíba, during the period from 1989 to 2018. It is an ecological approach, with a time series, quantitative and spatial. The data were extracted from online mortality atlas of the Instituto Nacional de Câncer José Alencar Gomes da Silva (INCA). The average number of deaths by GC in Paraíba was equivalent to 1145.5, with the highest number of deaths in the mesoregion of Mata Paraibana (2056) and in Agreste Paraibano (1358). All mesoregions showed a continuous increase in the average number of deaths over the years, with a greater increase in deaths in Borborema (4.5x). As for the types of GC, it was observed that the highest average of deaths was for cervical cancer (227.8) in Mata Paraibana and the lowest average of deaths occurred in the Borborema region, for vagina cancer (1.0). In all regions, uterine cancer deaths were more frequent, followed by Without Other Specification (WOS) uterus, ovary, body of the uterus, other genitals, vulva and vagina. We conclude that there is an increasing mortality due to GC throughout Paraíba, with heterogeneity between regions and types of cancers.

Key words: Epidemiology, mortality, women's health, neoplasms of female genitals. 


\section{Introdução}

A incidência e mortalidade por neoplasias vem aumentando em todo o mundo, sendo justificado em parte pela transição demográfica populacional e em decorrência de alterações no estilo de vida, como também pela mudança na distribuição e na prevalência dos fatores cancerígenos. O câncer representa um problema de saúde pública mundial, estando entre as quatros principais causas de morte antes dos 70 anos de idade, na maioria dos países (Pereira et al. 2015; Ferlay et al. 2018; INCA 2020a,b).

No século XXI, o câncer ganhou uma ampla visibilidade, visto que consiste em uma doença crônica degenerativa que afeta várias dimensões da vida humana causando impactos econômicos na sociedade, necessitando de tratamentos especializados, prolongados e onerosos (Pereira \& Santos 2020).

Em países em desenvolvimento, vem sendo verificado uma transição na incidência dos principais tipos de câncer, com um declínio das malignidades associadas a infecções, como câncer de boca e o câncer de vagina e o aumento das patologias relacionadas a melhores condições socioeconômicas, como a neoplasia de pulmão e o câncer de colo uterino, que são intrinsecamente associados à incorporação de hábitos e atitudes ligados à urbanização sedentarismo, alimentação inadequada, entre outros (Torres-Pereira et al. 2012; Bray et al. 2018).

Além disso, pode-se citar outras questões que contribuem para essa transição epidemiológica, como: idade, obesidade, história familiar, terapia hormonal, infecções sexualmente transmissíveis e baixo nível de escolaridade. Contudo, quando se refere ao câncer ginecológico, não existe um consenso sobre os aspectos associados, porém, a transmissão do Papilomavírus Humano (HPV) é mencionada na literatura como a responsável pelo risco aumentado de desenvolver algumas neoplasias do sistema reprodutor, como câncer de colo do útero, vagina e vulva (Sousa 2020).

Diante da diversidade de tipos de neoplasias, é perceptível que nas mulheres, os cânceres ginecológicos estão entre as enfermidades mais comuns. Dessa forma, são considerados um importante problema de saúde mundial. Essas patologias desenvolvem-se nos órgãos reprodutivos femininos, abrangendo o colo de útero, corpo do útero, ovários, endométrio, vagina e vulva (Moradi-Marjaneh et al. 2019).

Essas enfermidades do sistema reprodutor feminino, assim como a maioria das neoplasias malignas, consistem em doenças que podem ser prevenidas e curadas, quando diagnosticadas precocemente. No entanto, muitos fatores como baixo nível de escolaridade, insegurança, problemas econômicos e dificuldade de acesso aos serviços de saúde, são fatores que corroboram para que na maioria dos casos, as mulheres com cânceres ginecológicos sejam diagnosticadas em estágio avançado da doença, diminuindo as possibilidades de cura (Evcili \& Bekar 2020).

Mundialmente, esses cânceres são responsáveis por cerca de $10 \%$ de todos os tipos de malignidades diagnosticadas em mulheres. Além disso, estas malignidades diagnosticadas em mulheres, representam uma das principais causas de morte, mesmo com variação da origem anatômica do tumor primário, em diferentes regiões, embora existam diferenças na distribuição e frequências nos casos de mortalidade (Hailu et al. 2020). No Brasil, os tipos de cânceres que mais acometem as mulheres são o câncer de útero, endométrio e o de ovário, sendo a neoplasia de colo uterino a mais frequente na população feminina, e do endométrio o segundo mais comum. Contudo, é importante salientar que já existem estudos realizados nas mais diversas partes do país os quais mostram diferentes taxas de incidência e mortalidade para as neoplasias do trato reprodutor feminino (Sousa 2020).

Dentre as neoplasias ginecológicas, o câncer de colo uterino e o de ovário, são os que apresentam as maiores taxas de mortalidade. Em relação as regiões brasileiras, o Nordeste é o que apresenta os maiores índices de incrementos nas taxas de mortalidade pelos cânceres citados, com destaque para o estado da Paraíba, com perspectivas de aumento para as próximas décadas, tendo em vista, que apesar da possibilidade de detecção precoce por meio de rastreamento populacional de reconhecida sensibilidade, a maioria dos casos de cânceres ginecológicos são diagnosticados em estágios avançados ou metástase, o que contribuem, em 
parte, com os altos registros de mortalidades em decorrência da falta de investimento e políticas públicas que minimizem os fatores de riscos (Barbosa et al. 2015; Meira et al. 2019; Nascimento et al. 2020).

Diante do exposto, este estudo teve por objetivo analisar a distribuição do perfil de mortalidade de diferentes cânceres ginecológicos que acometem as mulheres em mesorregióes do estado da Paraíba, durante o período de trinta anos (1989 a 2018).

\section{Metodologia}

Trata-se de um estudo quantitativo e espacial, de modelo epidemiológico do tipo ecológico de série temporal. Segundo Borges et al. (2009), o delineamento epidemiológico do tipo ecológico é capaz de analisar comparativamente variáveis globais, permitindo correlação entre os indicadores de condições de vida e de situação de saúde.

Sendo assim, foi estudada e analisada a distribuição do perfil de mortalidade dos diferentes cânceres ginecológicos nas variadas mesorregiões do estado da Paraíba (Sertão Paraibano, Agreste Paraibano, Borborema e Mata Paraibana), durante o período de trinta anos (1989 a 2018). Os dados sobre os óbitos foram extraídos do Atlas On-line de Mortalidade por Câncer, disponibilizados no site do Instituto Nacional de Câncer José Alencar Gomes da Silva (INCA) do Ministério da Saúde, durante o mês de outubro de 2020. O Atlas sobre mortalidade utilizado possui como uma de suas finalidades auxiliar os profissionais de saúde pública na determinação de prioridades necessárias à prevenção e ao controle do câncer (INCA 2020b).

Os óbitos pelos cânceres ginecológicos para o período de 1989 a 2018 foram obtidos considerando as codificações (C51 - C57) da Classificação Estatística Internacional de Doenças e Problemas Relacionados à Saúde - Décima Revisão (CID-10), conforme mostra a Tabela 1. Além do tipo de câncer, foram consideradas as seguintes variáveis: ano e local da ocorrência do óbito, sexo e causa básica da morte.

Tabela 1. Classificação e nomeação dos tipos de cânceres ginecológicos segundo a Classificação Estatística Internacional de Doenças e Problemas Relacionados à Saúde - Décima Revisão (CID-10). *SOE: Sem Outra Especificação.

\begin{tabular}{c|c}
\hline Classificação CID-10 & Cânceres Ginecológicos \\
\hline C51 & Vulva \\
\hline C52 & Vagina \\
\hline C53 & Colo do útero \\
\hline C54 & Corpo do útero \\
\hline C55 & Útero, SOE* \\
\hline C56 & Ovário \\
\hline C57 & Outros órgãos genitais femininos, SOE* \\
\hline
\end{tabular}

Os dados utilizados foram inicialmente coletados como frequência relativa (percentual) por mesorregião e como valor absoluto por municípios, sendo este, agregado em microrregiões do estado da Paraíba e organizados no software Microsoft Excel 2010 e posteriormente, analisados no programa estatístico GraphPad Prism $5^{\circledast}$, no qual foi possível expressá-los na forma de média de número de óbitos por mesorregião com intervalo de confiança (IC).

Ressalta-se que o presente estudo utilizou uma fonte de dados de domínio público, sendo, portanto, dispensado do parecer do Comitê Ética de Pesquisa (CEP) obedecendo às normas éticas exigidas pela Resolução nº 466/2012 Conselho Nacional de Saúde (CNS).

\section{Resultados}

A análise de óbitos por cânceres ginecológicos no estado da Paraíba, durante o período de 1989 a 2018, é representada por uma média de 1145.5 óbitos, podendo variar de 281 a 2056 mortes entre as mesorregiões, em virtude de tamanho populacional, estilo de vida e acesso aos 
serviços de saúde. Na Figura 1, observa-se a peculiaridade de cada uma das mesorregiões do estado da Paraíba e percebe-se que os menores número de óbitos foram na região da Borborema (281) e do Sertão Paraibano (887), estando abaixo da média de óbitos do estado e os maiores números de óbitos foram encontrados na Mata Paraibana (2056) e no Agreste Paraibano (1358), com valores superiores à média de óbitos do estado.

Porém, ao analisar a média de óbitos considerando intervalos temporais, percebe-se que independente da variação em número de óbitos, todas as mesorregiões apresentaram um aumento de mortalidade com o decorrer dos anos, em que ocorre um aumento de $4 \mathrm{x}$ a média de morte no Sertão, com variação da média de 16.83 e 67.86, respectivamente, para o intervalo de 1989 a 1998 e 2009 a 2018 (Figura 1A); aumento de 3.3x a média de morte no Agreste, com variação da média de 28.71 e 95.25, respectivamente, para o intervalo de 1989 a 1998 e 2009 a 2018 (Figura 1B); aumento de 4.5x a média de morte na Borborema, com variação da média de 9.0 e 40.0, respectivamente, para o intervalo de 1989 a 1998 e 2009 a 2018 (Figura 1C) e aumento de 3x o número de mortes na Mata, com variação da média de 95.0 e 275.5, respectivamente, para o intervalo de 1989 a 1998 e 2009 a 2018 (Figura 1D).

Pode-se, então, afirmar que apesar da Borborema ser a região com o menor número de óbitos, esta também foi a mesorregião com maior crescimento na média de óbitos durante os últimos 30 anos, enquanto a região da Mata, que dispõe do maior número de óbitos, foi a mesorregião com menor crescimento da média de mortes, no período estudado.
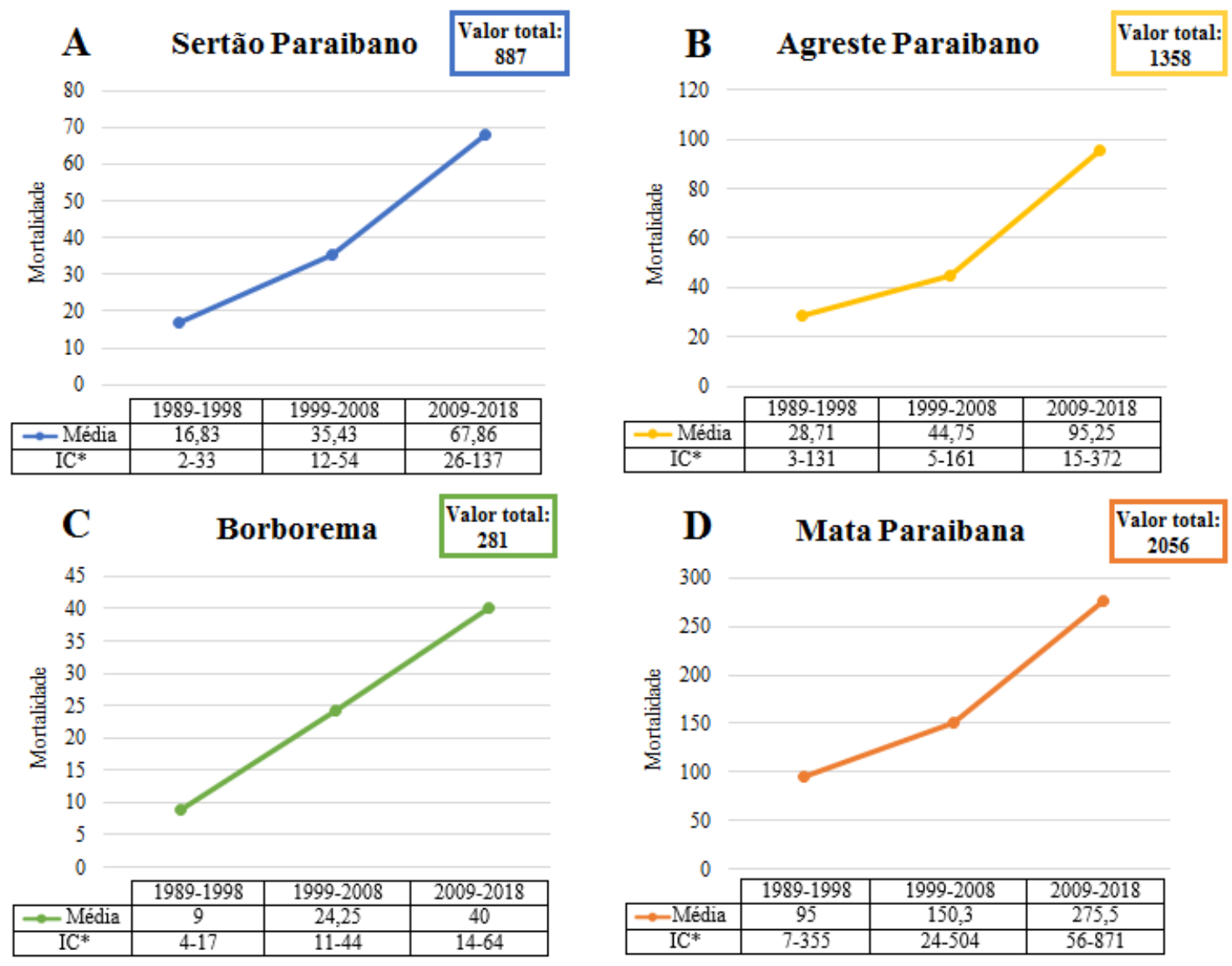

Figura 1. Mortalidade por cânceres ginecológicos no (A) Sertão Paraibano, (B) Agreste Paraibano, (C) Borborema e (D) Mata Paraibana, durante o período de 1989 a 2018. Os valores foram expressos na forma de média, com intervalo de confiança. *IC: Intervalo de Confiança.

Uma vez observado o aumento da mortalidade por cânceres ginecológicos na Paraíba, ao longo do período estudado, foi avaliado a distribuição dos óbitos dentro da diversidade de neoplasias ginecológicas, considerando as mesmas regiões e o mesmo período temporal. Percebe-se que a neoplasia de colo do útero apresentou as maiores médias de mortes nas quatro 


\section{Cânceres ginecológicos na Paraíba}

mesorregiões, sendo a Mata Paraibana responsável pelos maiores registros (227.8), seguida do Sertão Paraibano (104.5), Agreste Paraibano (83.2) e Borborema (33.2).

Referente à neoplasia do útero, verificou-se um aumento nos valores das médias de mortalidade em todas as mesorregiões, com a Mata Paraibana representando a localidade com maior valor da média (126.8), acompanhada do Sertão Paraibano (31.6), Agreste Paraibano (27.9) e Borborema (16.0).

As quatro mesorregiões também apresentaram um expressivo número de mortalidade por câncer de ovário nos períodos analisados. A maior média de mortalidade foi evidenciada na mesorregião da Mata Paraibana com 104.5 (IC: 13-372), seguido do Agreste Paraibano 26.4 (IC: 3-112), Sertão Paraibano 18.9 (IC: 3-45) e Borborema 8.0 (IC: 2-15).

Em contrapartida, as menores médias foram registradas para as neoplasias de corpo do útero (4.8) na mesorregião do Sertão Paraibano, vagina (1.0) na Borborema e vulva (1.7) no Agreste Paraibano (Tabela 2).

Tabela 2. Distribuição da mortalidade por cânceres ginecológicos de acordo com as mesorregiões do Estado da Paraíba, durante o período de trinta anos, 1989-2018. IC: Intervalo de Confiança. *SOE: Sem Outra Especificação.

\begin{tabular}{|c|c|c|c|c|c|}
\hline \multirow[b]{2}{*}{ Cânceres Ginecológicos } & \multicolumn{5}{|c|}{ Mesorregiões do Estado da Paraíba } \\
\hline & $\begin{array}{l}\text { Médias } \\
\text { (IC) }\end{array}$ & Agreste Paraibano & Borborema & Mata Paraibana & Sertão Paraibano \\
\hline C51 - Vulva & $\begin{array}{l}\text { Média } \\
\text { (IC) }\end{array}$ & $\begin{array}{c}1.7 \\
(1-4)\end{array}$ & $\begin{array}{c}2.0 \\
(1-3)\end{array}$ & $\begin{array}{c}9.2 \\
(2-31)\end{array}$ & $\begin{array}{c}1.8 \\
(1-3)\end{array}$ \\
\hline C52 - Vagina & $\begin{array}{l}\text { Média } \\
\text { (IC) }\end{array}$ & $\begin{array}{c}2.1 \\
(1-5)\end{array}$ & 1.0 & $\begin{array}{c}5.0 \\
(1-14)\end{array}$ & $\begin{array}{c}2.0 \\
(1-4)\end{array}$ \\
\hline C53 - Colo do útero & $\begin{array}{l}\text { Média } \\
\text { (IC) }\end{array}$ & $\begin{array}{c}83.2 \\
(11-313)\end{array}$ & $\begin{array}{c}33.2 \\
(14-55)\end{array}$ & $\begin{array}{c}227.8 \\
(43-737)\end{array}$ & $\begin{array}{c}104.5 \\
(26-64)\end{array}$ \\
\hline C54 - Corpo do útero & $\begin{array}{l}\text { Média } \\
\text { (IC) }\end{array}$ & $\begin{array}{c}13.4 \\
(2-41)\end{array}$ & $\begin{array}{c}7.0 \\
(2-11)\end{array}$ & $\begin{array}{c}35.0 \\
(5-123)\end{array}$ & $\begin{array}{c}4.8 \\
(2-8)\end{array}$ \\
\hline C55 - Útero, SOE* & $\begin{array}{l}\text { Média } \\
\text { (IC) }\end{array}$ & $\begin{array}{c}27.9 \\
(4-111)\end{array}$ & $\begin{array}{c}16.0 \\
(8-32)\end{array}$ & $\begin{array}{c}126.8 \\
(15-432)\end{array}$ & $\begin{array}{c}31.6 \\
(6-56)\end{array}$ \\
\hline C56 - Ovário & $\begin{array}{l}\text { Média } \\
\text { (IC) }\end{array}$ & $\begin{array}{c}26.4 \\
(3-112)\end{array}$ & $\begin{array}{c}8.0 \\
(2-15)\end{array}$ & $\begin{array}{c}104.5 \\
(13-372)\end{array}$ & $\begin{array}{c}18.9 \\
(3-45)\end{array}$ \\
\hline $\begin{array}{lll}\text { C57 - Outros } & \text { órgãos } \\
\text { genitais, } & \text { SOE* } & \\
\end{array}$ & $\begin{array}{l}\text { Média } \\
\text { (IC) }\end{array}$ & $\begin{array}{c}13.6 \\
(2-82)\end{array}$ & $\begin{array}{c}4.5 \\
(1-10)\end{array}$ & $\begin{array}{c}12.5 \\
(4-21)\end{array}$ & $\begin{array}{c}5.7 \\
(5-6)\end{array}$ \\
\hline
\end{tabular}

Além disso, foi possível verificar, neste estudo, o valor da frequência relativa no que diz respeito a mortalidade pelos cânceres ginecológicos. Assim, de acordo com o Sertão Paraibano, verifica-se que o câncer de colo de útero foi a neoplasia com maior destaque (42.91), seguida da neoplasia de útero (29.51), ovário (17.78), corpo do útero (4.38), outros órgãos genitais (2.98), vagina (1.29) e vulga (1.16) (Figura 2A).

Em relação ao Agreste Paraibano notou-se que os valores são semelhantes, porém com um aumento na mortalidade por câncer de colo de útero (50.3) e outros órgãos genitais (8.18) e diminuição do câncer de útero (16.74), ovário (15.84), corpo do útero (7.06), vulva (1.13) e vagina (0.75) (Figura 2B).

A mesorregião Borborema, por sua vez, apresentou elevados índices de mortalidade por câncer de colo de útero (42.91), útero (22.88) e vulva (3.33), com diminuições consideráveis entre o câncer de ovário (10.7), corpo do útero (9.96), outros órgãos genitais (6.27) e vagina (0.37) quando comparado com as mesorregiões mencionadas (Figura 2C).

Referente à Mata Paraibana, vê-se, por fim, que o câncer de colo de útero é o que apresenta a maior mortalidade (44.77), seguido do câncer de útero (24.64), ovário (20.31), corpo do útero (6.8), vulva (1.8), outros órgãos genitais (1.21) e vagina (0.97) (Figura 2D).

Pontua-se, portanto, que as neoplasias com os maiores registros de percentual nas quatro mesorregiões foram colo uterino, útero e ovário. Em contrapartida, as menores taxas de 
percentual foram registradas entre as neoplasias de vagina, vulva, outros órgãos genitais feminino e corpo do útero.

A
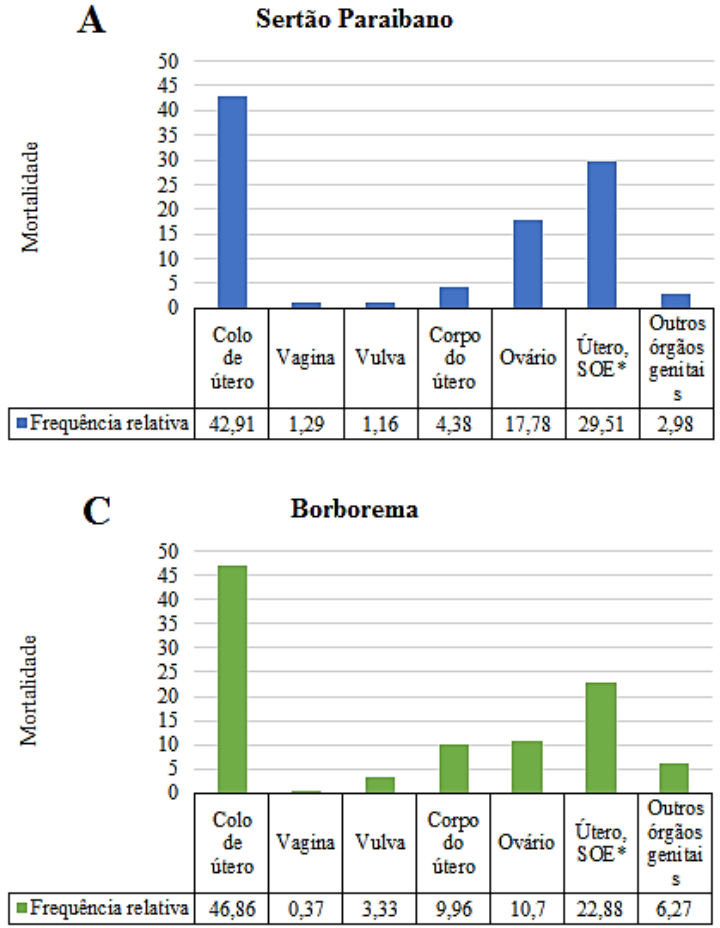

B
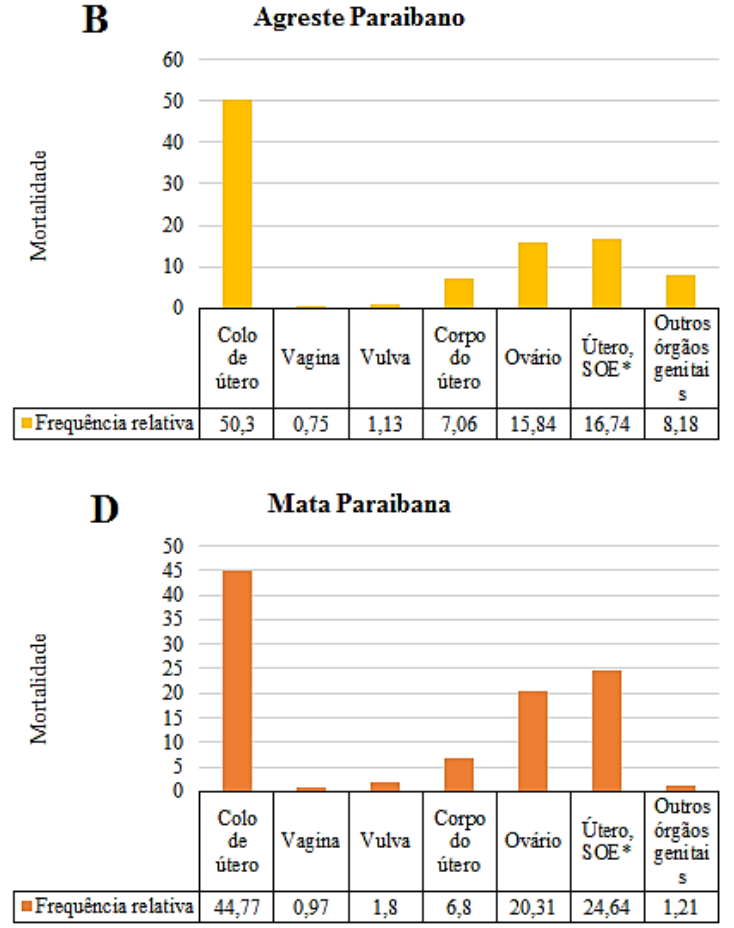

Figura 2. Distribuição da mortalidade por cânceres ginecológicos no (A) Sertão Paraibano, (B) Agreste Paraibano, (C) Borborema e (D) Mata Paraibana, durante o período de trinta anos, 1989 a 2018. Os valores foram expressos na forma frequência relativa. *SOE: Sem outra especificação.

\section{Discussão}

Os resultados apresentados neste estudo buscaram enfatizar o avanço dos principais tipos de cânceres ginecológicos nas mesorregiões do estado da Paraíba. Nossos achados demonstraram que, independente de mesorregião avaliada, observou-se um incremento no número de casos de mortes por cânceres ao longo das décadas, corroborando com o estudo realizado por Kemo et al. (2019), em que se verificou o mesmo comportamento ao longo dos anos para os tipos de cânceres de mama e colo uterino.

Para este estudo, é possível destacar que as maiores médias foram registradas na última década (2009 a 2018), o que explica em parte os argumentos apresentados por Duarte (2016), ao afirmar que o aumento no número de casos, entre décadas, pode estar relacionado à transição demográfica que as regiões do país vêm apresentando, com tendência de redução nos casos de doenças infectocontagiosas e aumento no número de casos de doenças crônicas, tais como o câncer.

Quanto à diferença da média de óbitos por neoplasias ginecológicas entre regiões, é importante salientar que cada localidade possui uma densidade populacional diferente. Além disso, questões como padrões de acesso aos serviços de saúde, principalmente os de diagnósticos iniciais, são realizados de forma diferenciada e irregular entre os estados brasileiros. Certamente, aspectos sociais e de desenvolvimento econômico também corroboram para que algumas regiões apresentem diferenças entre os índices de mortalidade por câncer (Kemo et al. 2019).

O ritmo do avanço da mortalidade por neoplasias ginecológicas que se observa no estudo, não aponta para uma redução ou estabilização, mas para uma ascensão das mortes nos próximos anos, se continuar seguindo os mesmos comportamentos que os anos anteriores, o que se torna um fator preocupante, haja vista a existência de políticas de prevenção. Na presente pesquisa, observa-se um aumento das médias nas quatros mesorregiões analisadas, com destaque para a 
Borborema e o Sertão Paraibano. Portanto, esses dados demandam atuações mais contundentes para frear o avanço das mortes pela doença (Carvalho \& Paes 2019).

Esse aumento no número de óbitos que foi verificado no presente estudo, está de acordo com os trabalhos realizados por Barbosa et al. (2015), quando se refere a neoplasia de corpo do útero, em que na região do Nordeste do Brasil, essa malignidade vem apresentado aumento na taxa de incidência e mortalidade, bem como acréscimo significativo de acordo com a taxa de progressão para o ano 2030.

As neoplasias da vulva e vagina apresentaram as menores médias de óbitos durante o período estudado. Apesar de serem considerados tipos de cânceres raros, essas malignidades contribuem significativamente para as taxas de morbidade no mundo, desta forma, também é pertinente discutir sobre prevenção e controle de todas as causas de óbitos por cânceres ginecológicos, independente da frequência. Torna-se importante salientar que essas malignidades quando diagnosticadas em mulheres mais jovens, etiologicamente possuem associação com o câncer de colo do útero, especificamente relacionado à persistência de infecções por HPV de alto risco (Esteve et al. 2016; Adams \& Cuello 2018).

Para os diferentes tipos de câncer, observou-se uma elevada mortalidade nas mesorregiões para a malignidade de colo uterino. Essa afirmação é condizente com os estudos realizados por Paul et al. (2016), em que essa neoplasia apresentou uma frequência equivalente a 81.20\%, durante o período de 2005 a 2014, na República de Camarões-África.

Esses resultados presentes em nosso contexto, referente à mortalidade pela neoplasia de colo do útero podem ser explicados pelos múltiplos fatores de risco para essa patologia, incluindo o fato de muitas mulheres jovens serem infectadas com vários tipos de vírus do papiloma humano, que consiste na principal etiologia do câncer cervical, tendo em vista que dos mais de 100 tipos de HPV existentes, aproximadamente 40 afetam o trato genital humano, e desses, pelo menos 13 tipos são considerados oncogênicos, apresentando um risco aumentando a probabilidade de provocar infecções persistentes e estar associados a lesões precursoras, sendo os tipos 16 e 18 os mais associados com o câncer cervical em nível mundial (Kemo et al. 2019; Simões \& Zanusso Junior 2019).

Outros fatores contribuintes para os elevados índices de óbitos por câncer de colo uterino consiste na ausência de vacina contra esse vírus, múltiplos parceiros sexuais, a prática de relações sexuais precoce ou relações desprotegidas, tendo em vista que o uso de preservativo consiste no método mais eficaz e acessível para a prevenção de contaminação por infecções sexualmente transmissíveis, incluindo o HPV (Kemo et al. 2019; Petry et al. 2019).

A neoplasia ginecológica de colo uterino pode ser diagnostica em estágios iniciais quando ocorre a realização do exame Papanicolau que é realizado de forma gratuita pelo Sistema Único de Saúde (SUS), e serve como método preventivo. Porém, os elevados números de óbitos podem ser explicados pela não realização do exame, consequentemente, contribui para o diagnóstico tardio elevando o risco de morte. A falta de conhecimento está discutida na literatura como um dos motivos pelas quais as mulheres não realizam o exame, como também representações da vulnerabilidade envolvidas na realização do procedimento e dos sentimentos de dor, medo, receio, desconforto, nervosismo e vergonha diante do profissional (Costa et al. 2018).

Referente a esses valores elevados da mortalidade por neoplasias ginecológicas, convém destacar que nas regiões do Brasil, vem sendo observado um envelhecimento populacional, consequentemente, a mortalidade por doenças crônicas vem aumentando (Bray \& Soerjomataram 2015). Além disso, as localidades com baixos Índices de Desenvolvimento Humano (IDH) estão mais expostas aos cânceres associados à infecção. No Brasil, observou-se uma predominância das taxas de mortalidade por câncer de colo do útero nesses locais. Nas regiões com o IDH mais elevado, verifica-se a presença mais frequente de neoplasias relacionados aos hábitos e estilos de vida (Guimarães et al. 2016).

De acordo com os resultados apresentados neste estudo, verifica-se que a neoplasia de ovário apresenta um baixo percentual equivalente à média da mortalidade quando comparado à neoplasia de colo uterino. Esses achados estão de acordo com os observados por Barbosa et al. (2015), ao verificar que apesar da neoplasia de ovário apresentar baixos números de óbitos, ao 
longo dos anos vem ocorrendo um aumento considerável referente à frequência da mortalidade, tendo em vista que é caracterizada como uma das mais letais, devido ser assintomática, e estar relacionada com o diagnostico tardio e a diminuição da taxa de sobrevida. Contudo, diferem com os dados que foram obtidos em outros estudos, em que essa patologia foi registrada como o segundo tipo de câncer mais prevalente nos órgãos reprodutores femininos (Maheshwari et al. 2016; Hailu et al. 2020).

No presente estudo, constata-se, ainda, que há uma heterogeneidade da média da mortalidade nas diferentes mesorregiões e nos diferentes tipos de neoplasias ginecológicas estudadas. De acordo com os estudos feitos por Camargo (2019), as regiões Norte e Nordeste, mantiveram-se com as maiores proporções de registros de mortalidade, com destaque para o estado da Paraíba, responsável por apresentar os maiores percentuais de incrementos na média de mortalidade. Essa realidade se associa às dificuldades de acesso aos serviços de saúde e à má qualidade na assistência prestada (Lima \& Queiroz 2014).

Por isso, reforça-se a necessidade de retificação dos registros a fim de evitar subnoticações, já que estes dados são responsáveis por viabilizar estudos e análises territoriais para estratégias de enfrentamento.

\section{Conclusões}

Conclui-se com este estudo, que, independente de mesorregião avaliada, observou-se um incremento considerável na média de mortalidade por cânceres ginecológicos, conforme avanços dos períodos anuais, com destaque para a região da Mata Paraibana que apresentou maiores valores e da mesorregião da Borborema com os menores valores.

Observou-se ainda uma concentração das mortes por câncer de colo do útero, útero, ovário, corpo do útero, vulva, vagina e outros órgãos genitais femininos, com destaque para a neoplasia de colo uterino e útero, que foram as malignidades responsáveis pelas maiores médias de óbitos no estudo.

Diante disso, espera-se que este estudo possa contribuir para a realização de planejamentos de saúde e do seu acesso, haja vista a detecção de diferenças de mortalidade por cânceres ginecológicos entre as regiões da Paraíba, que podem ser reflexos de como os serviços de saúde foram e estão sendo organizados. Além disso, servir como instrumentos de monitoramento do estado de saúde da população residente em cada localidade da Paraíba, com o objetivo de amenizar o quadro atual, as medidas de planejamento e as políticas de saúde.

É imprescindível, portanto, acompanhar o número e a evolução dos casos em cada uma destas localidades, como também a necessidade de mais estudos que permitam avaliar o perfil de mortalidade de cada um desses cânceres ao longo dos anos, nas diferentes mesorregiões, considerando a taxa ajustada e a acessibilidade aos serviços de diagnóstico precoce.

\section{Referências}

Adams T.S. \& Cuello M.A. (2018) Cancer of the vagina. International Journal of Gynecology \& Obstetrics, 143(2): 14-21. https://doi.org/10.1002/ijgo.12610

Barbosa I.R, Costa I.C.C, Pérez M.M.B. \& Souza D.L.B. (2015) Tendências e Projeções da Mortalidade pelos Cânceres Específicos ao Gênero no Brasil. Tese (Programa de Pósgraduação em Saúde Coletiva). Universidade Federal do Rio Grande do Norte, Centro de Ciências da Saúde, Natal, Rio Grande do Norte.

Borges D.M.L., Sena M.F., Ferreira M.A.F. \& Roncalli A.G. (2009) Mortalidade por câncer de boca e condição sócio-econômica no Brasil. Cadernos de Saúde Pública, 25(2): 321-327. https://doi.org/10.1590/S0102-311X2009000200010

Bray F. \& Soerjomataram I. (2015) The changing global burden of cancer: transitions in human development and implications for cancer prevention and control (p. 5-25). In: Gelband H., Bank W., Sankaranarayanan R., Jha P. \& Horton S. (Eds). Disease control priorities: Cancer. $3^{\circ}$ 
edition. Washington: World Bank Publications. 363 p. https://doi.org/10.1596/978-1-4648-03499_ch2

Bray F., Ferlay J., Soerjomataram I., Siegel R.L., Torre L.A. \& Jemail A. (2018) Global cancer statistics 2018: GLOBOCAN estimates of incidence and mortality worldwide for 36 cancers in 185 countries. CA: A Cancer Journal for Clinicians, 68(6): 394-424. https://doi.org/10.3322/caac.21492

Camargo J.D.A.S. (2019) Evolução temporal da mortalidade por câncer de mama nos estados da Região Nordeste sob a perspectiva dos efeitos idade, período e coorte. Dissertação (Programa de Pós-graduação em Demografia). Universidade Federal do Rio Grande do Norte, Centro de Ciências Exatas e da Terra, Natal, Rio Grande do Norte.

Carvalho J.B. \& Paes N.A. (2019) Desigualdades socioeconômicas na mortalidade por câncer de mama em microrregiões do Nordeste brasileiro. Revista Brasileira Saúde Materna Infantil, 19(2): 401-410. https://doi.org/10.1590/1806-93042019000200008

Costa R.S.L., Silva M.V.R. \& Souza T.N. (2018) Fatores que levam a não adesão ao exame preventivo do câncer do colo uterino em uma unidade de saúde do Acre em 2014. DêCiência em Foco, 2(2): 5-18.

Duarte D.A.P. (2016) Iniquidade social e câncer em mulheres: análise da Mortalidade por Câncer de Mama e Colo do Útero nas Microrregiões de Saúde de Minas Gerais no Período de 20082012. Dissertação (Programa de Pós-Graduação em Saúde Coletiva). Universidade Federal de Juiz de Fora, Juiz de Fora, Minas Gerais.

Esteve A.B., Planell C.D.C. \& Marqués F.M.P. (2016) Cáncer de vulva em lamujerjoven no asociado a infección por virusdel papiloma humano: a propósito de um caso. Clínica $e$ Investigación em Ginecología y Obstetrícia, 43(2): 89-91. https://doi.org/10.1016/j.gine.2014.12.014

Evcili F. \& Bekar M. (2020) Prevention of gynecological cancers: the affecting factors and knowledge levels of Turkish women. Journal of Health Research, 34(5): 431-441. https://doi.org/10.1108/JHR-07-2019-0171

Ferlay J., Colombet M., Soerjomataram I., Mathers C., Parkin D.M., Piñeros M., Znaor A. \& Bray F. (2018) Estimating the global cancer incidence and mortality in 2018: GLOBOCAN sources and methods. International Journal of Cancer, 144(8): 1941-1953. https://doi.org/10.1002/ijc.31937

Guimarães R.M., Muzi C.D., Teixeira M.P. \& Pinheiro S.S. (2016) A transição da mortalidade por cânceres no Brasil e a tomada de decisão estratégica nas políticas públicas de saúde da mulher. Revista de Políticas Públicas, 20(1): 33-50. https://doi.org/10.18764/2178-2865.v20n1p35-50

Hailu H.E., Mondul A.M., Rozek L.S. \& Geleta T. (2020) Descriptive Epidemiology of breast and gynecological cancers among patients attending Saint Paul's Hospital Millennium Medical College, Ethiopia. PLoS ONE, 15(3): 1-11. https://doi.org/10.1371/journal.pone.0230625

INCA (2020a) Ministério da Saúde. Instituto Nacional de Câncer José Alencar Gomes da Silva. Estimativa 2020. Rio de Janeiro: INCA.

INCA (2020b) Ministério da Saúde. Instituto Nacional de Câncer José Alencar Gomes da Silva (INCA). Atlas on-line de mortalidade. Rio de Janeiro: INCA.

Kemo A.D.D., Nkegoum B., Nangue C., Djuikwo F., Bita'a L.B. \& Tebeu P.M. (2019) Histoepidemiological of gynecological and breast cancers at the University Teaching Hospital of Yaoundé. The Pan African Medical Journal, 33(130): 1-10. https://doi.org/10.11604/pamj.2019.33.130.18874

Lima E.E.C. \& Queiroz B.L. (2014) Evolution of the deaths registry system in Brazil: associations with changes in the mortality profile, under-registration of death counts, and ill-defined causes of death. Cadernos de Saúde Pública, 30(8): 1721-1730. https://doi.org/10.1590/0102-311X00131113

Maheshwari A., Kumar N. \& Mahantshetty U. (2016) Gynecological cancers: A summary of published Indian data. South Asian Journal of Cancer, 5(3): 112-120.

https://doi.org/10.4103/2278-330X.187575 


\section{Cânceres ginecológicos na Paraíba}

Meira K.C., Santos J., Silva C.M.F.P., Ferreira A.A., Guimarães R.M. \& Simões T.C. (2019) Efeitos da idade-período e coorte na mortalidade por câncer do ovário no Brasil e suas grandes regiões. Cadernos de Saúde Pública, 35(3): 1-15. https://doi.org/10.1590/0102-311x00087018

Moradi-Marjaneh R., Hassanian S.M., Hasanzadeh M., Rezayi M., Maftouh M., Mehramiz M., Ferns G.A., Khazaei M. \& Avan A. (2019) Therapeutic potential of toll-like receptors in treatment of gynecological cancers. IUBMB Life, 71(5): 549-564.

https://doi.org/10.1002/iub.2011

Nascimento G.S., Silva A.S., Oliveira C.B.S. \& Dantas B.B. (2020) Taxa de Mortalidade por Câncer de Ovário na Região Nordeste do Brasil (p 1084-1101). In: One G.M.C. \& Porto M.L.S. (Eds). Saúde a Serviço da Vida. Volume 2. João Pessoa: IDEA. 1140 p.

Paul E.N.J., Charlotte T.N., Jules O.M.L., Godefroy S., Daniel E.S.J., Michelle E.F.A., Louis E.O.J. \& Alan H. (2016) Epidemiological and Histological Profile of Cervical Cancer in Cameroon: About 2078 Cases. Open Journal of Obstetrics and Gynecology, 6(4): 1-8. https://doi.org/10.4236/ojog.2016.64029

Pereira B.E. \& Santos E.S.B.T (2020) Consumo de carne vermelha e processada associados a oncologia: a importância do acompanhamento e intervenção nutricional. Monografia (Bacharelado em Nutrição). Faculdade de Ciências da Educação e Saúde, Centro Universitário de Brasília, Brasília.

Pereira D.S., Nogueira J.A.D. \& Silva C.A.B. (2015) Qualidade de vida e situação de saúde de idosos: um estudo de base populacional no Sertão Central do Ceará. Revista Brasileira de Geriatria e Gerontologia, 18(4): 893-908. https://doi.org/10.1590/1809-9823.2015.14123

Petry S., Padilha M.I., Kuhnen A.E. \& Meirelles B.H.S. (2019) Saberes de estudantes de enfermagem sobre a prevenção de Infecções Sexualmente Transmissíveis. Revista Brasileira de Enfermagem, 72(5): 1145-1152. https://doi.org/10.1590/0034-7167-2017-0801

Simões L.P. \& Zanusso Junior G. (2019) Vírus hpv e o desenvolvimento de câncer de colo de útero - uma revisão bibliográfica. Revista Uningá, 56(1): 98-107.

Sousa J.D. (2020) Prevalência global de câncer ginecológico desafios no diagnóstico e tratamento. Monografia (Programa de Pós-Graduação em Aplicações Complementares às Ciências Militares). Escola de Saúde do Exército, Rio de Janeiro.

Torres-Pereira C.C., Angelim-Dias A., Melo N.S., Lemos Jr. C.A. \& Oliveira E.M.F. (2012) Abordagem do câncer da boca: uma estratégia para os níveis primário e secundário de atenção em saúde. Caderno de Saúde Pública, 28: 30-39. http://dx.doi.org/10.1590/S0102311X2012001300005 